\title{
Strain Test Performance of Brillouin Optical Time Domain Analysis and Fiber Bragg Grating Based on Calibration Test
}

\author{
Dingding Zhang, ${ }^{1,2}$ Wengang Du, ${ }^{1,2 *}$ Jing Chai, ${ }^{1}{ }^{2}$ and Wulin Lei ${ }^{1}$ \\ ${ }^{1}$ College of Energy Engineering, Xi'an University of Science and Technology, Xi'an 710054, China \\ ${ }^{2}$ Xi'an University of Science and Technology, Ministry of Education of the Western Mining and Mine Disaster \\ Prevention and Control of Key Laboratory, Xi'an 710054, China
}

(Received January 5, 2021; accepted March 22, 2021)

Keywords: BOTDA, FBG, three-dimensional digital speckle, uniform-strength beam, strain coefficient

Optical fiber sensing technology provides a new method for the deformation monitoring of engineering structures. To verify the reliability and accuracy of distributed optical fiber sensing technology based on Brillouin scattering, i.e., Brillouin optical time domain analysis (BOTDA) and fiber Bragg grating (FBG) technologies for deformation monitoring, the strain test performances of BOTDA and FBG technologies were investigated by carrying out calibration tests on a uniform-strength beam and uniaxial compression tests on standard rock specimens. The performances of a strain gauge, three-dimensional digital speckle measurement based on a digital image correlation (DIC) matching technique, and distributed optical fiber and FBG sensors were comparatively analyzed, and the strain measurement performance, monitoring stability, system error, and strain coefficients of distributed optical fibers with different diameters were obtained. Auxiliary verification was carried out by ANSYS numerical simulation. The measurement accuracy of the distributed optical fibers was found to be $\pm 25 \mu \varepsilon$. When the frequency shift of an optical fiber was positive, the fiber was under tensile stress, and when the frequency shift was negative, the fiber was under compressive stress. The fluctuation of DIC measurement data was greater than that obtained with the FBG sensor, which indicates that FBG measurement is more stable. The maximum fluctuation rates of the strain gauge data and FBG data were 0.092 and 0.031 , respectively. The stability of FBG technology for strain measurement is clearly better than that of traditional resistance strain gauges. A thicker packaging material increased the contact area between the optical fiber and the object under test, made the stress transfer more efficient, and improved the sensitivity of the optical fiber. The strain coefficients calculated by numerical simulation were larger than the calibration test results. By comprehensively considering the two sets of results, the strain coefficients of optical fibers with diameters of $0.1,0.7$, and $0.9 \mathrm{~mm}$ were found to be $0.0455,0.0485$, and $0.0520 \mathrm{MHz} /$ $\mu \varepsilon$, respectively. Research on the basic test performance of optical fiber sensing technology is of great significance for promoting its more effective application in engineering fields.

*Corresponding author: e-mail: 403870017@qq.com

https://doi.org/10.18494/SAM.2021.3255 


\section{Introduction}

In underground or above-ground geotechnical engineering structures such as mine shafts, roadways, slopes, dams, and foundation pits, the monitoring of internal deformation is an important part of ensuring safety. Deformation monitoring is generally embodied in the monitoring of the stress, strain, and displacement of the structural body, where macrodisplacement monitoring is the easiest to achieve. Stress and strain are internal deformation parameters caused by changes in external parameters (load and temperature). Therefore, strain is an important parameter for characterizing structural deformation. In recent years, strain monitoring has developed from traditional resistance strain gauges and vibrating string strain gauges to fiber Bragg gratings (FBGs) and the latest distributed optical fiber sensing techniques, i.e., Brillouin optical time domain analysis (BOTDA), thus realizing a leap from discrete monitoring to distributed monitoring. In a large civil engineering structure, a discrete monitoring pattern cannot easily delineate the strain field distribution on a large scale. ${ }^{(1)}$ If an optical fiber sensor network is used, the structure can be monitored in all directions and more deformation information can be obtained.

FBG technology has been widely used in engineering and scientific research. ${ }^{(2-6)}$ It is a mature technology in the field of optical fiber sensing. It can be made into different geometric shapes according to requirements, and has been widely used in strain and temperature measurement in various fields. Distributed optical fiber sensing technology based on the Brillouin scattering principle (BOFS) takes a light wave as the carrier and an optical fiber as the medium. Sensing optical fibers, similarly to a nervous system, can not only sense external information but also transmit information, so as to integrate sensing and transmission. Compared with traditional monitoring methods, BOFS has the advantages of electromagnetic insulation, water and moisture resistance, intrinsic safety, high accuracy, and distributed measurement. These characteristics make BOFS attractive for detecting the deformation of large structures, such as coal mining overburden, as well as roadway convergent deformation, where the strain field is expected to be heterogeneous. Since Horiguchi et al. ${ }^{(7)}$ and Culverhouse et al. ${ }^{(8)}$ first used the Brillouin scattering effect in an optical fiber for distributed temperature and strain measurements, extensive theoretical and experimental research has been conducted. ${ }^{(9-15)}$ This effect has been used for monitoring oil and gas pipelines, ${ }^{(16)}$ concrete and steel bridges, ${ }^{(17,18)}$ composite structures, ${ }^{(19)}$ dams, and slopes, and for landslide stability assessment. ${ }^{(20)}$

The premise of monitoring using ordinary single-mode communication optical fibers is that the basic parameters of the fibers are known. The basic parameters of optical fibers produced by different manufacturers vary considerably due to variations in the production process, doping materials, and sheath materials. Therefore, optical fiber sensors need to be calibrated before monitoring. In this paper, a uniform-strength beam test was designed for verifying the strain test performance, and the calibration strain coefficient was obtained by comparison with theoretical calculation results, strain gauge monitoring values, FBG monitoring values, and ANSYS numerical calculation results. 


\section{Basic Principles}

\subsection{Concept of uniform-strength beam}

In a uniform-strength beam, the maximum normal stress on each cross section of the beam is equal. To make the stress of each section equal, the cross-section size of the beam should be changed according to the bending moment to ensure that the strength of the beam at different positions is the same. As shown in Fig. 1, let the beam thickness be $h$, the beam length be $L$, the cross-section width of the fixed end be $b$, the distance from the loading end to an arbitrary cross section be $x$, and the width of the arbitrary cross section be $b_{x}$. The cross-section width of the beam varies linearly along the axis direction, that is,

$$
b_{x} / x=b / L
$$

For a uniform-strength beam subjected to vertical downward stress, the part above the neutral surface is under tensile stress and the part below the neutral surface is under compressive stress. The maximum tensile stress is located at the upper edge of the cross section. For a solid rectangular cross-section beam, the inertia moment $I_{z}$ and the bending section coefficient $W_{z}$ are

$$
\begin{aligned}
& I_{z}=\int_{A} y^{2} d A=\frac{b_{x} h^{3}}{12}, \\
& W_{z}=\frac{I_{z}}{\left|y_{\max }\right|}=\frac{1}{6} b_{x} h^{2} .
\end{aligned}
$$

The maximum principal stress of the beam is
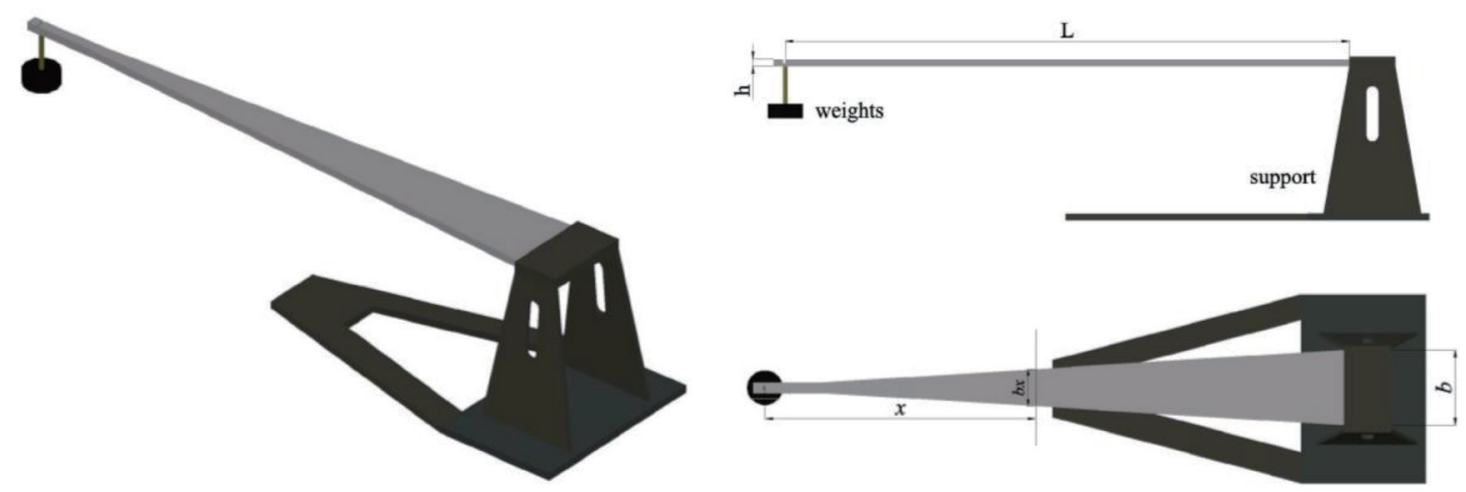

Fig. 1. Views of uniform-strength beam. $L$ is the length of the beam, $h$ is the thickness, $b_{x}$ is the width of an arbitrary cross section, $x$ is the distance between the cross section and the loading end, and $b$ is the width of the fixed end. 


$$
\sigma_{\max }=\frac{M(x)}{W_{z}(x)}=\frac{6 F \cdot x}{b_{x} h^{2}}=\frac{6 F \cdot L}{b \cdot h^{2}} .
$$

Equation (4) shows that the maximum principal stress on a cross section is only related to the load $F$ and not to the position of the cross section, that is, the stress on each cross section is equal under a constant load. Therefore, because the thickness of the beam is constant, to ensure that the principal stress remains constant, $x$ and $b_{x}$ must increase simultaneously, that is, the cross section of the beam should gradually increase in the direction of the beam support. According to the theory of elasticity, the strain of the beam is

$$
\varepsilon=\frac{\sigma}{E}=\frac{6 F x}{E b_{x} h^{2}}=\frac{6 F \cdot L}{E b h^{2}}
$$

Through this formula, the theoretical strain of the beam under a load can be obtained. On the basis of this principle, the sensing fiber can be calibrated.

\subsection{Principle of BOTDA and FBG}

In BOTDA technology, which is based on the Brillouin scattering principle, pulsed light and continuous light are injected at the two ends of a complete fiber, and a stimulated Brillouin amplification effect occurs when the frequency difference between the two lights is equal to the Brillouin frequency shift in a specific region of the fiber. Figure 2 shows the working principle of BOTDA. The frequency shift has a linear relationship with the strain and temperature at each location of the optical fiber. Therefore, by detecting the Brillouin frequency continuously, the strain and temperature distributions along the fiber can be obtained.

During measurement, the frequency of the two lasers is adjusted continuously to detect the power at the receiving end, and the frequency difference at the moment of maximum energy transfer is determined as follows:

$$
\begin{gathered}
\Delta V_{B}=V_{B(\varepsilon)}-V_{B(0)}=C_{1} \Delta T+C_{2} \Delta \varepsilon, \\
\varepsilon=\left[\Delta V_{B}-C_{1} \Delta T\right] / C_{2},
\end{gathered}
$$

where $\Delta V_{B}$ is the change in the Brillouin frequency shift, $C_{1}$ is the sensitivity coefficient of the fiber to temperature, $V_{B(\varepsilon)}$ is the strain-induced Brillouin shift, $C_{2}$ is the sensitivity coefficient of the fiber to strain, and $V_{B(0)}$ is the initial Brillouin frequency shift. The strain and temperature of the optical fiber are obtained by detecting the frequency shift variation of the Brillouin signal and the variation in the normalized signal power to achieve distributed measurement.

An FBG sensor changes the wavelength of the reflected light wave according to changes in the ambient temperature and strain. If internal writing or a laser is used to form periodic defects on the fiber to change the refractive index of the core region, when the external parameters (temperature, stress) change, the refractive index of the grating will also change, causing the 


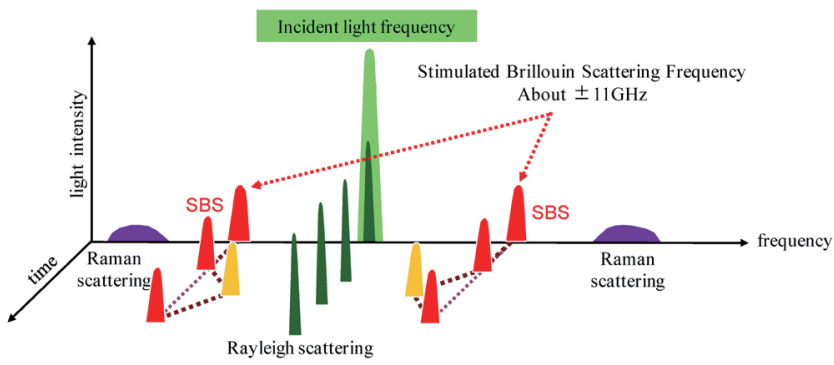

(a)

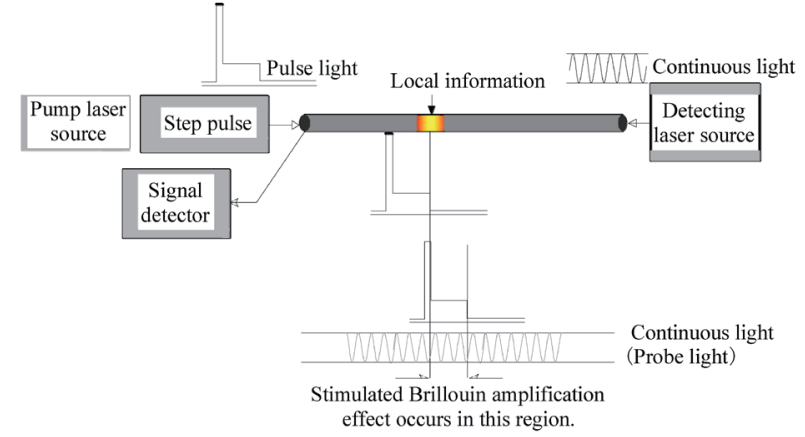

(b)

Fig. 2. (Color online) Working principle of BOTDA. The stimulated Brillouin amplification effect occurs when the optical fiber is subjected to stress and temperature changes. (a) Propagation pattern of light in an optical fiber. (b) Principle of BOTDA.

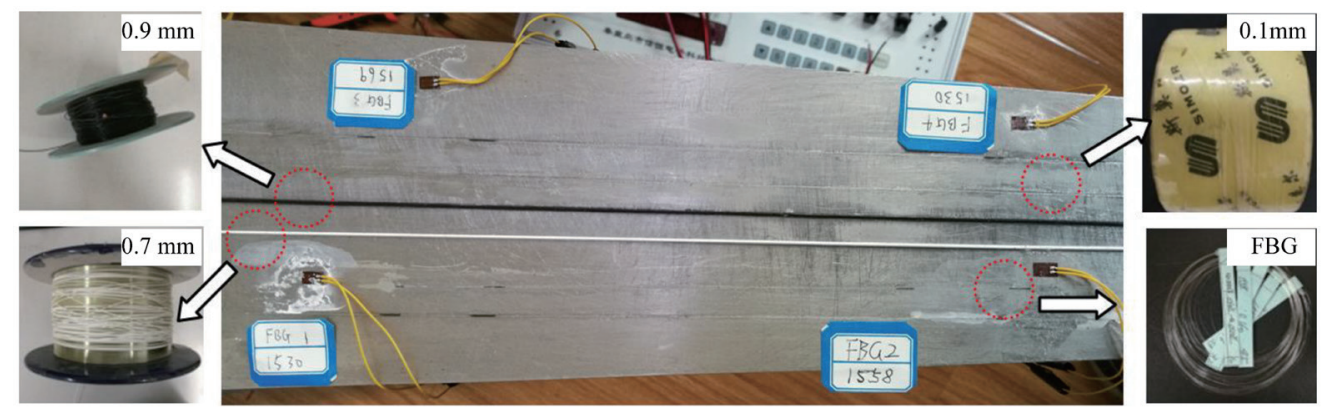

Fig. 3. (Color online) Layout of sensors in calibration test. Four strain gauges were pasted beside the four FBG sensors on the upper surface, and two strain gauges were pasted beside the two FBG sensors on the lower surface. The diameters of the black, white, and transparent optical fibers were $0.9,0.7$, and $0.1 \mathrm{~mm}$, respectively.

wavelength of the sensor to drift. The change in the wavelength $\Delta \lambda_{B}$ is obtained by detecting the wavelength drift, temperature, and strain, which satisfy the following relationship:

$$
\Delta \lambda_{B}=K_{\varepsilon} \Delta \varepsilon_{g}+K_{T} \Delta T
$$

where $\Delta \varepsilon_{g}$ is the strain, $\Delta T$ is the temperature, $K_{\varepsilon}$ is the strain calibration coefficient of the sensor, and $K_{T}$ is the temperature calibration coefficient of the sensor.

Most sensors including resistive strain gauges, FBG sensors, and distributed optical fiber sensors have temperature and strain dual sensitivity characteristics. If the temperature significantly changes the measured parameters (resistance, center wavelength, center frequency, etc.), temperature compensation should be used to eliminate the temperature effect. The Brillouin frequency shift of a distributed optical fiber has the dual sensitivity characteristics of strain and temperature. Therefore, when the strain is measured, the frequency shift caused by the temperature effect should be eliminated. When the temperature change is less than $5{ }^{\circ} \mathrm{C}$, the 
influence of the temperature effect can be neglected. ${ }^{(21,22)}$ Without considering the temperature, the Brillouin frequency shift only has a linear relationship with strain. Therefore, it is crucial to obtain the coefficient between the Brillouin frequency shift and the strain. The calibration test to obtain the strain coefficient will be introduced below.

\section{Calibration Test}

\subsection{Layout of sensors}

The sensors used in the test were strain gauges, single-mode compact-jacketed optical fibers with diameters of $0.1,0.7$, and $0.9 \mathrm{~mm}$, and FBG sensors with central wavelengths of 1530, 1558, and $1569 \mathrm{~nm}$. The testing equipment comprised an NBX-6055 PPP-BOTDA analyzer, an SM125 FBG demodulator, and a CM-1L static resistance strain analyzer. Six strain gauges were used in the test. Four strain gauges (gauges 1-4) were arranged on the upper surface of the beam and two strain gauges (gauges 5 and 6) were arranged on the lower surface. The FBG sensors were placed next to the strain gauges. Five distributed optical fiber sensors with a length of $1 \mathrm{~m}$ were arranged on the surface of the uniform-strength beam. Three optical fibers with diameters of 0.1 , 0.7 , and $0.9 \mathrm{~mm}$ (optical fibers $1-3$, respectively) were arranged on the upper surface, and those with diameters of 0.9 and $0.1 \mathrm{~mm}$ (optical fibers 4 and 5, respectively) were arranged on the lower surface. The three optical fibers on the upper surface were fused to form a complete loop and were positioned by the water bath heating method. The optical fibers were fixed on the beam surface by epoxy resin adhesive.

\subsection{Positions of distributed optical fibers}

To simplify the measurement system and shorten the measurement time, several parts of the optical fibers were usually connected into a complete loop. For this reason, we needed to know the specific position of each part of the fibers in the loop. Because the Brillouin frequency shift is sensitive to both strain and temperature, fibers were usually positioned by heating them at specific positions. As shown in Fig. 4, we used a water bath for heating at points T1, T2, and T3. As the temperature increased, the instrument was able to detect the peak of the center frequency at each point, revealing the spatial positions of points T1, T2, and T3 in the fiber loop. Segments $\mathrm{AB}, \mathrm{CD}$, and EF, each with a length of $1 \mathrm{~m}$, were the parts of the fiber used for effective measurement. The length was used to calculate the spatial positions of the effective measurement fibers in the loop. The spatial positions of optical fibers 1, 2, and 3 were 2.633-3.633 m, 4.94-5.94 $\mathrm{m}$, and 7.193-8.193 $\mathrm{m}$ and the spatial positions of optical fibers 4 and 5 on the lower surface were $1.65-2.65 \mathrm{~m}$ and $1.77-2.77 \mathrm{~m}$, respectively.

\section{Results and Analysis}

\subsection{Systematic measurement error in BODTA}

Repeated measurements of a free-segment optical fiber, which is not affected by external force and temperature changes, were carried out five times to determine the systematic 


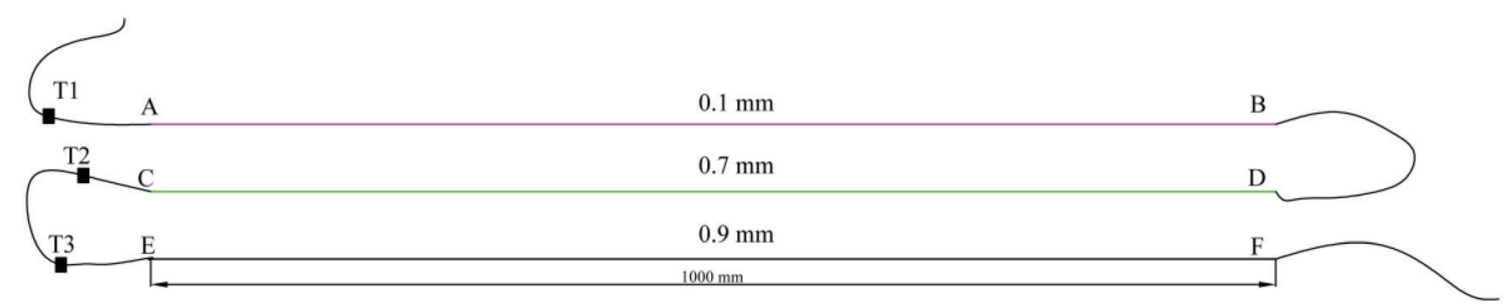

Fig. 4. (Color online) Distribution of temperature measurement points on distributed optical fiber. A water bath was placed at each black square to heat the optical fibers, allowing the change in the Brillouin frequency shift to be detected.

measurement error of the equipment. The abscissa in Fig. 5 is the spatial distribution of the optical fibers. Optical fibers with lengths of 2-25 m were selected, which included the tested fibers. The ordinate in Fig. 5 is the center frequency of the fiber. The results of the five tests were basically the same, which shows that the equipment worked stably and the system error was small. Taking the first measured center frequency as the reference value, the frequency shift caused by the system error was obtained as the difference between the last four measured results and the first one, and then the strain error was obtained from the strain coefficients (see Sect. 4.3). According to the graph, except for some abnormal data points, the measurement strain error caused by the stability of the system was within $25 \mu \varepsilon$. That is, when the measured strain is less than $25 \mu \varepsilon$, the measured data is invalid and cannot be used to analyze practical problems. When the measured strain exceeds $25 \mu \varepsilon$, the strain is considered to be caused by the deformation of the measured object. Compared with other strain measurement methods, the accuracy of strain measurement by the BOTDA technique is relatively high.

\subsection{FBG strain measurement performance}

FBG technology is relatively mature in the field of optical fiber sensing technology. Its main characteristics are high precision and sensitivity. To verify the accuracy and reliability of strain measurement using an FBG sensor, three-dimensional digital speckle technology was used for comparison. Digital speckle measurement based on a digital image correlation matching technique (DIC) is a photogrammetric technology that can measure the displacement of a whole field and the strain on an object surface with high precision. This technique has gradually been replacing traditional displacement measurement methods in recent years.

Figure 6 shows the strain measurement by the FBG sensor, strain gauge, and DIC. The specimen shown in Fig. 6(a) was made of barite powder, iron sand, sand, gypsum, alcohol, and rosin in a certain proportion. The specimen was allowed to dry naturally for one week after demolding and then cut into standard rock specimens. Strain gauges and FBG sensors were arranged along the radial and circumferential directions of the rock specimens, respectively. Artificial speckle was fabricated by spraying a layer of white primer on the surface of specimens, followed by black speckle to ensure a uniform and random distribution of speckle particles as shown in Fig. 6(b). Figure 6(c) shows the loading mode of the specimens; the uniaxial compression test of the specimens was conducted on an MTS universal testing machine. 


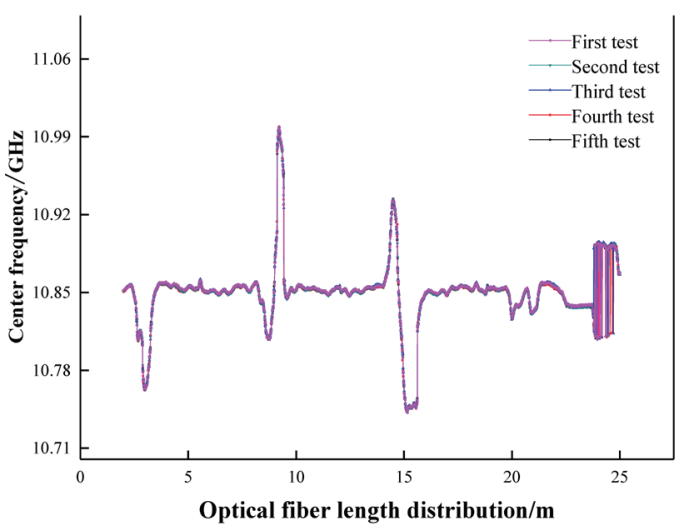

(a)

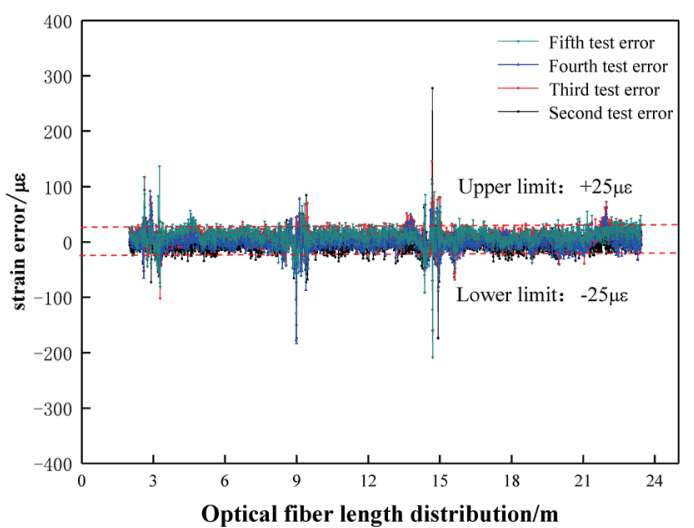

(b)

Fig. 5. (Color online) Accuracy test of distributed optical fiber. (a) Center frequency of the optical fiber, which is not affected by external forces and temperature variation. (b) System measurement error calculated with the reference value in the first test.

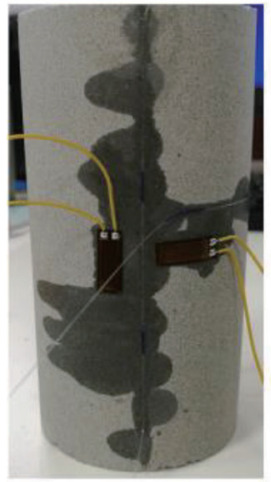

(a)

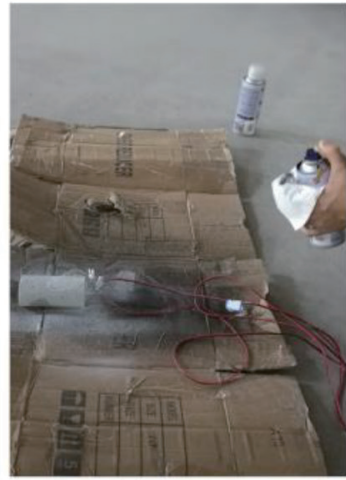

(b)

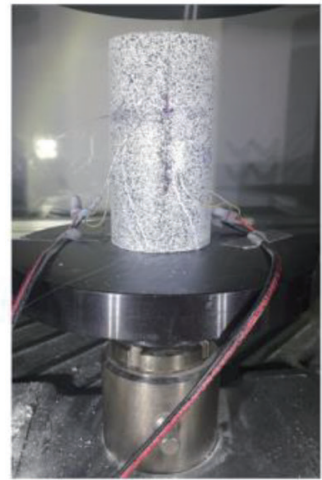

(c)

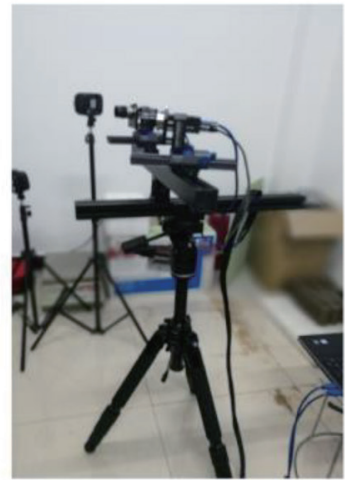

(d)

Fig. 6. (Color online) Test to compare strain measurement between FBG sensor and DIC technique. (a) Sensor layout pattern, (b) speckle fabrication, (c) loading mode of the specimens, and (d) DIC measurement system.

The relationship between the axial strain and time for the specimens detected by the FBG sensor and DIC is shown in Fig. 7. The trend of the strain measured by the two methods is similar, especially during elastic compression. The fluctuation of the DIC measurement data is greater than that of the FBG sensor data, which indicates that the FBG measurement is more stable. During the compression of specimen 25-1, the axial strain measured by the FBG sensor and DIC had the same trend with time. Near 70 s, both the FBG sensor and DIC indicated a peak strain, but the peak strain measured by DIC was larger than that measured by the FBG sensor. The axial strain of specimen 1-2 had the same trend: the peak strain was similar for both methods but the peak strain measured by DIC showed hysteresis. In this process, the strain measured by the strain gauge did not exceed $500 \mu \varepsilon$. Because the strain gauge was made of metal and was rectangular and planar, it had little contact with the cylindrical rock specimens during their deformation. Therefore, the measurement results were seriously distorted. The results of this test show that FBG has higher measurement accuracy. 

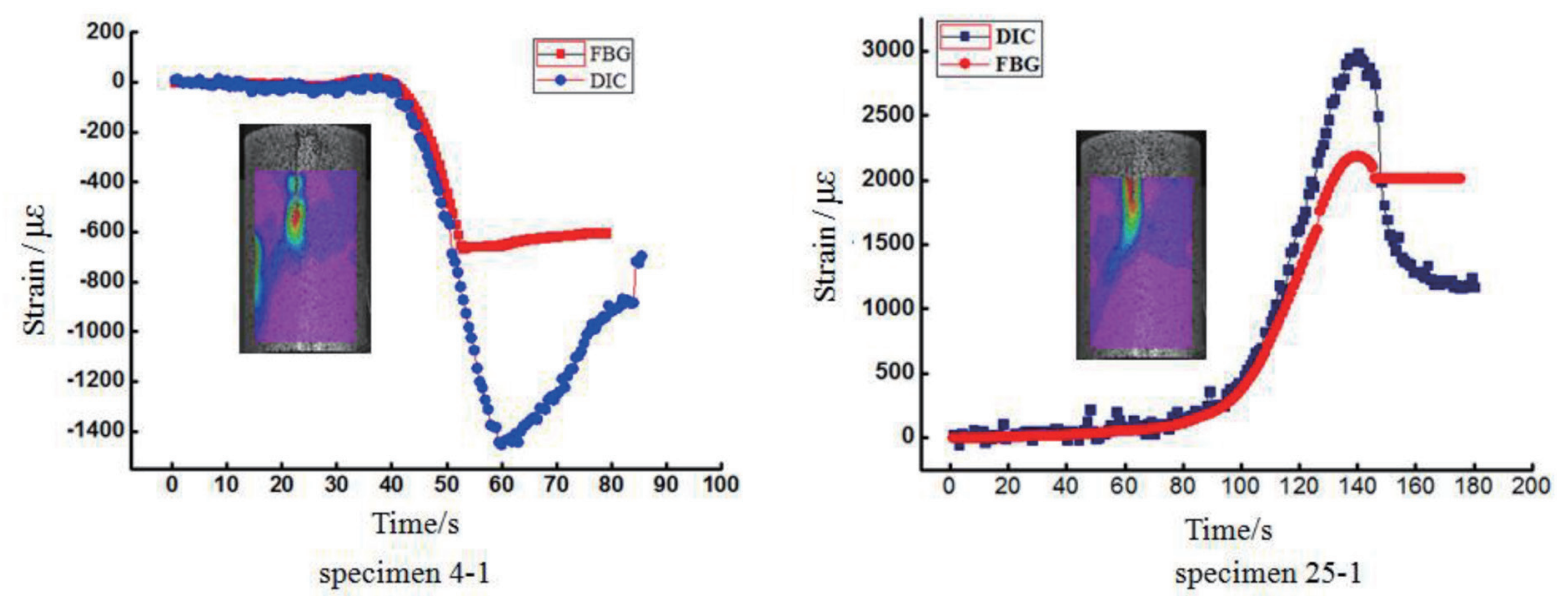

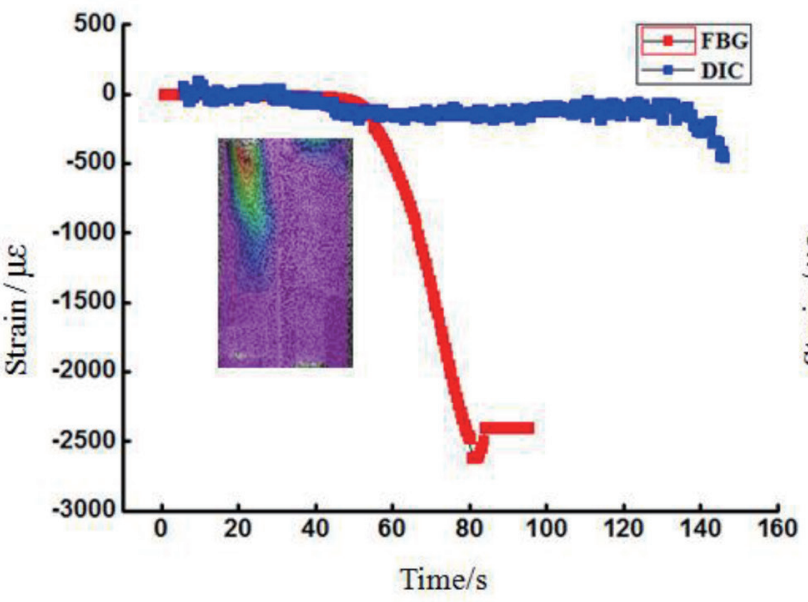

specimen 1-1

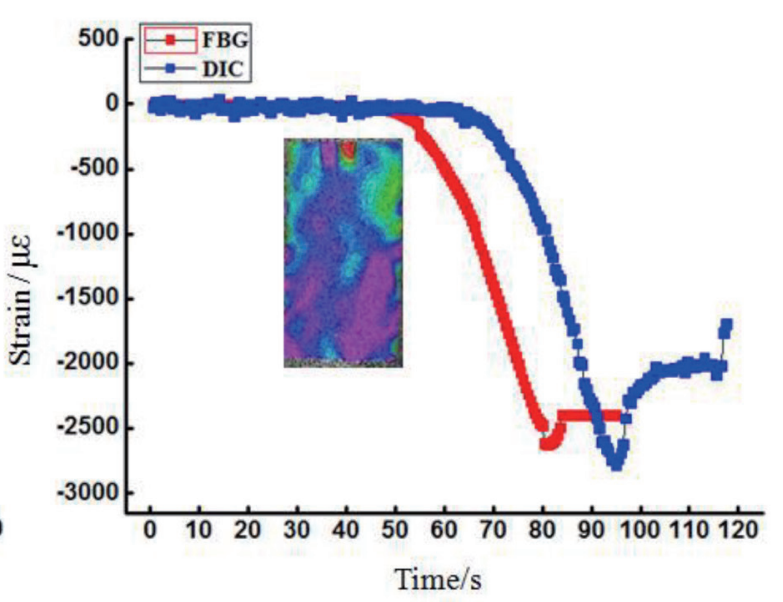

specimen 1-2

Fig. 7. (Color online) Relationship between axial strain and time for specimens measured by FBG sensor and DIC.

\subsection{Optical fiber strain coefficient}

The strain coefficient of the optical fiber is crucial when using BOTDA technology for deformation monitoring. Figure 8 shows the Brillouin frequency shift of different optical fiber sensors under different loads. The Brillouin frequency shift of the optical fibers on the upper surface of the uniform-strength beam was positive. The frequency shift of each load gradient was basically constant and increased with increasing load. Owing to the influence of environmental noise and the system error, the frequency shifts of the optical fibers fluctuated in a small range. The frequency shift of the optical fiber on the lower surface of the uniformstrength beam was negative, and the variation of the frequency shift was basically consistent with that of the optical fibers on the upper surface. According to material mechanics theory, when the two ends of a beam are bent downward, tensile stress acts on the upper part of the neutral plane and compressive stress acts on the lower part of the neutral plane. Therefore, when the frequency shift of the fiber is positive, the fiber is under tensile stress, and when the 


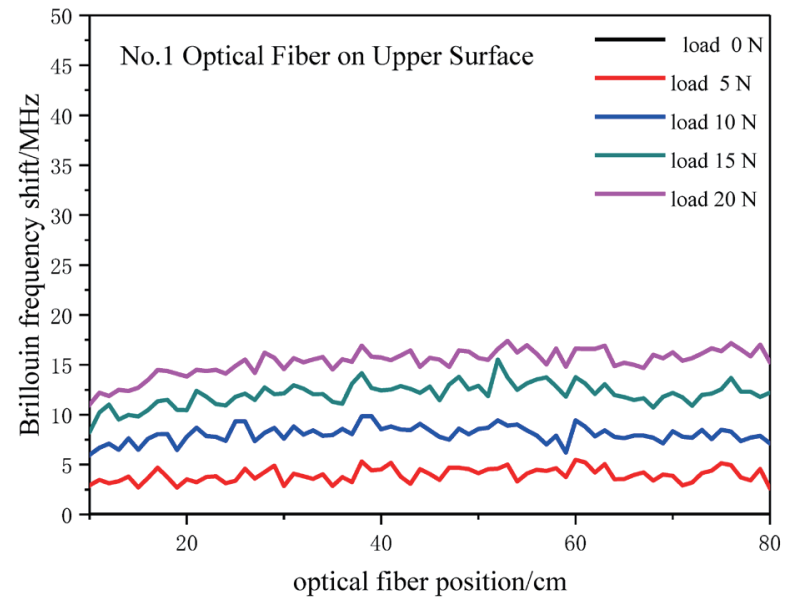

(a)

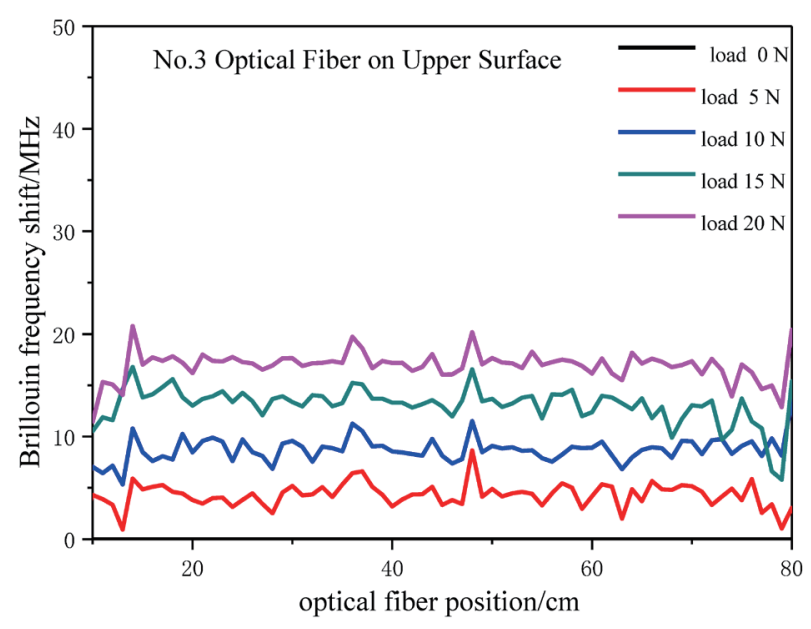

(c)

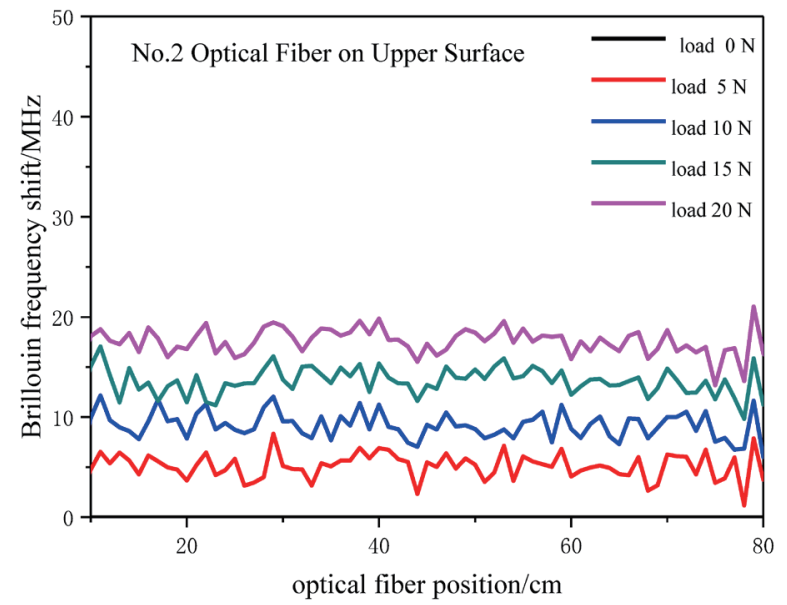

(b)

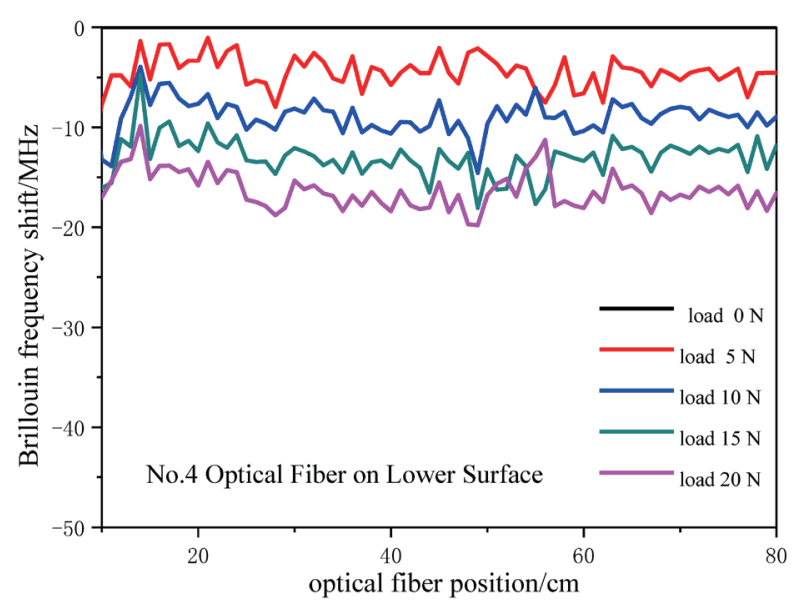

(d)

Fig. 8. (Color online) Brillouin frequency shift detected by BOTDA. Optical fibers 1, 2, and 3 were located on the upper surface of the uniform-strength beam and had diameters of $0.1,0.7$, and $0.9 \mathrm{~mm}$, respectively, and optical fiber 4 was located on the lower surface and had a diameter of $0.9 \mathrm{~mm}$.

frequency shift is negative, the fiber is under compressive stress. On the basis of this rule, the stress state of the measured object can be characterized using a distributed optical fiber.

It can also be seen from Fig. 8 that when the same load was applied, among the optical fibers on the upper surface of the beam, the frequency shift of optical fiber 3 with $0.9 \mathrm{~mm}$ diameter, was the largest, followed by optical fiber 2 with $0.7 \mathrm{~mm}$ diameter, then optical fiber 1 with 0.1 $\mathrm{mm}$ diameter. Thus, the greater the diameter of the optical fiber, the greater the frequency shift under the same load and the more sensitive the optical fiber is to the load. The frequency shifts of optical fibers 3 and 4 on the upper and lower surfaces, respectively, were basically the same under the same load but the former were positive and the latter were negative. The core diameter of each bare optical fiber was basically the same (about $0.1 \mu \mathrm{m}$ ). To improve the strength of optical fibers to prevent brittle fracture and enhance their sensitivity, polyimide and polyurethane are usually used to encapsulate optical fibers. According to the calibration test result, the thicker 
the packaging material, the greater the sensitivity to stress. A thicker packaging material increases the contact area between the optical fiber and the object under test, making the stress transfer more efficient and improving the sensitivity of the optical fiber.

The theoretical strain under different loads was calculated using Eq. (5). Assuming a beam length of $1.2 \mathrm{~m}$, a thickness of $0.005 \mathrm{~m}$, a cross-section width of the fixed end of $0.18 \mathrm{~m}$, and an elasticity modulus of $210 \mathrm{GPa}$, the theoretical strains under loads of 5, 10, 15, and $20 \mathrm{~N}$ were 96, 192, 288, and $384 \mu \varepsilon$, respectively. For optical fiber 1 with a diameter of $0.1 \mathrm{~mm}$, the average Brillouin frequency shifts under loads of $5,10,15$, and $20 \mathrm{~N}$ were 4.334, 8.350, 12.933, and $15.940 \mathrm{MHz}$, respectively, and the calculated strain coefficient was $0.0413 \mathrm{MHz} / \mu \varepsilon$. For optical fiber 2 with a diameter of $0.7 \mathrm{~mm}$, the average Brillouin frequency shifts under loads of 5, 10, 15, and $20 \mathrm{~N}$ were $5.324,9.009,13.999$, and $17.756 \mathrm{MHz}$, respectively, and the calculated strain coefficient was $0.0441 \mathrm{MHz} / \mu \varepsilon$. For optical fiber 3 with a diameter of $0.9 \mathrm{~mm}$, the average Brillouin frequency shifts under loads of $5,10,15$, and $20 \mathrm{~N}$ were $6.254,10.965,15.276$, and $19.867 \mathrm{MHz}$, respectively, and the calculated strain coefficient was $0.0471 \mathrm{MHz} / \mu \varepsilon$. The above data show that the strain coefficients of the optical fiber sensors were slightly different. Using the strain coefficients obtained by the calibration test, the Brillouin frequency shift can be transformed into the strain distribution of the measured object.

\subsection{Comparison of strain measurement performance of different sensors}

\subsubsection{Strain gauge measurement results}

The strain of each strain gauge before loading was $0 \mu \varepsilon$. After loading of $5 \mathrm{~N}$, the average strains of the four strain gauges on the upper surface and the two strain gauges on the lower surface were 88.5 and $-67 \mu \varepsilon$; after loading of $10 \mathrm{~N}$, they were 177.25 and $-132 \mu \varepsilon$; after loading of $15 \mathrm{~N}$, they were 266 and $-197.5 \mu \varepsilon$; and after loading of $20 \mathrm{~N}$, they were 338.75 and $-261.5 \mu \varepsilon$, respectively (Table 1). The strain of the upper surface was positive and that of the lower surface was negative, which indicates that tensile stress acted on the upper surface of the beam and compressive stress acted on the lower surface of the beam. This is consistent with the above results for distributed optical fiber detection.

As shown in Fig. 9, the fitting equation for the upper surface was $y=0.8+17.84 x$ with coefficient of determination $R^{2}=0.998$ and that for the lower surface was $y=-13.5 x-0.8$ with $R^{2}=0.999$. The strain gauge monitoring value had a strong linear relationship with the load. The

Table 1

Test results of uniform-strength beam calibration using strain gauges.

\begin{tabular}{lcccc}
\hline Strain gauge number & Load $5 \mathrm{~N}$ & Load $10 \mathrm{~N}$ & Load $15 \mathrm{~N}$ & Load $20 \mathrm{~N}$ \\
\hline 1 & 88 & 181 & 274 & 353 \\
2 & 93 & 183 & 271 & 312 \\
3 & 88 & 175 & 265 & 349 \\
4 & 85 & 170 & 254 & 341 \\
5 & -71 & -138 & -204 & -271 \\
6 & -63 & -126 & -191 & -252 \\
\hline
\end{tabular}




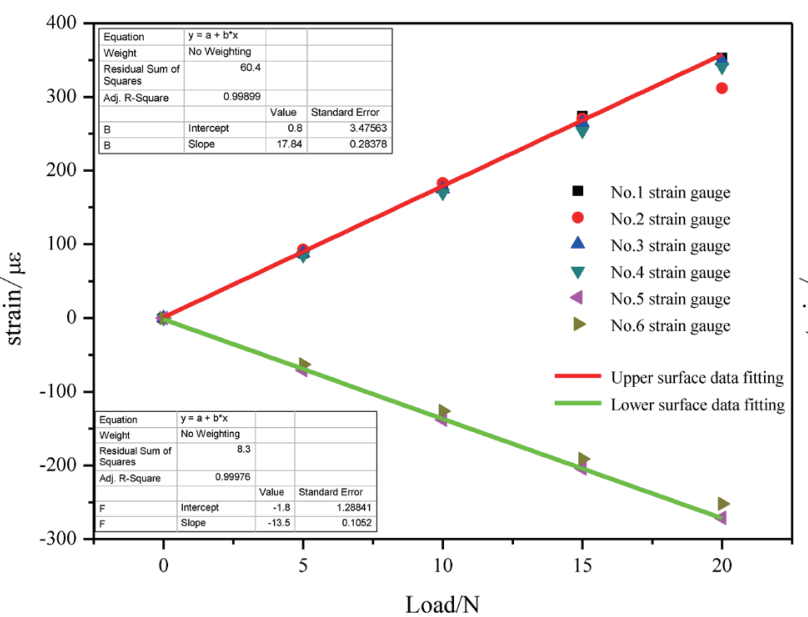

(a)

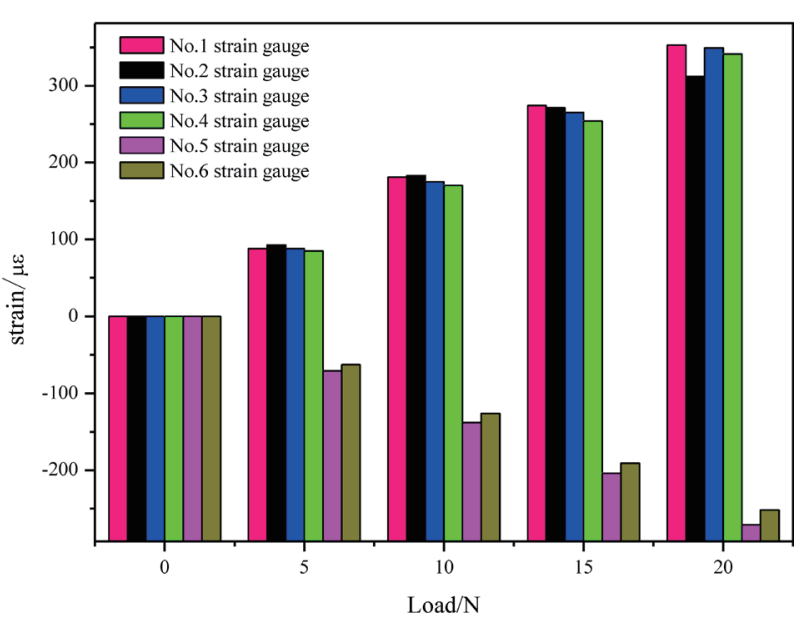

(b)

Fig. 9. (Color online) Statistical chart of strain gauge measurements. The strain had a strong linear relationship with the load, the strain of the upper surface was positive, and that of the lower surface was negative.

histogram in Fig. 9 shows that the strain at different positions on the same surface of the beam was approximately constant under the same load. However, the measured data fluctuated due to instrumental stability. To reflect the amount of fluctuation of the data, $k$ was defined as the maximum fluctuation rate of the data,

$$
k=\frac{1}{n} \sum_{1}^{n}\left(M_{j \max }-M_{j \min }\right) / M_{j E},
$$

where $M_{j \max }$ is the maximum in the set of data, $M_{j \min }$ is the minimum in the set of data, $M_{j E}$ is the average value, $1 \leq j \leq n$, and $n$ is the total number of loadings.

According to the above formula, the fluctuation rates of the strain under 5, 10, 15, and $20 \mathrm{~N}$ loading were $0.09,0.07,0.07$, and 0.12 , respectively, and the average value was 0.092 . From the above analysis, the strain gauge measurement results verify that the strain was constant at different positions of the uniform-strength beam.

\subsubsection{FBG measurement results}

Four FBG sensors were arranged on the upper surface of the beam at the same locations as the strain gauges. An FBG demodulator directly measured the central wavelength of the grating in a specific state (stress, temperature), and the strain was obtained using Eq. (8). The strain coefficient of the bare FBG was $0.845 \mu \varepsilon / \mathrm{pm}$. Table 2 shows the strain values obtained by the FBG sensors.

It can be seen from Fig. 10 that the stability of the FBG strain measurement was excellent and the fluctuation of the data was very small. The fitting equation of the strain measured by the FBG sensors was $y=1.07+15.85 x$ with fitting index $R^{2}=0.999$. The strain monitored by the FBG sensors had a strong linear relationship with the load, with linearity greater than that for the strain gauges. Similarly, Eq. (9) was used to calculate the maximum fluctuation rate of the 
Table 2

Test results of uniform-strength beam calibration using FBG sensors.

\begin{tabular}{lccccc}
\hline Serial number & Strain initial value $/ \mu \varepsilon$ & Load 5 N & Load 10 N & Load 15 N & Load 20 N \\
\hline FBG1 & 0 & 80.35955 & 160.23735 & 241.54325 & 315.6075 \\
FBG2 & 0 & 81.45647 & 163.7068 & 247.69715 & 324.86255 \\
FBG3 & 0 & 82.48045 & 163.5751 & 244.9317 & 322.46045 \\
FBG4 & 0 & 82.63255 & 165.9242 & 248.25255 & 326.2376 \\
\hline
\end{tabular}

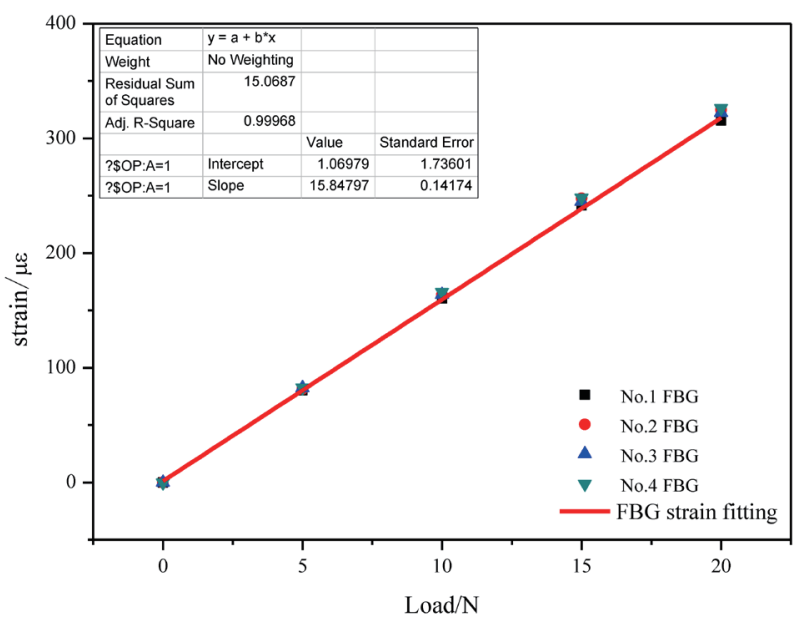

(a)

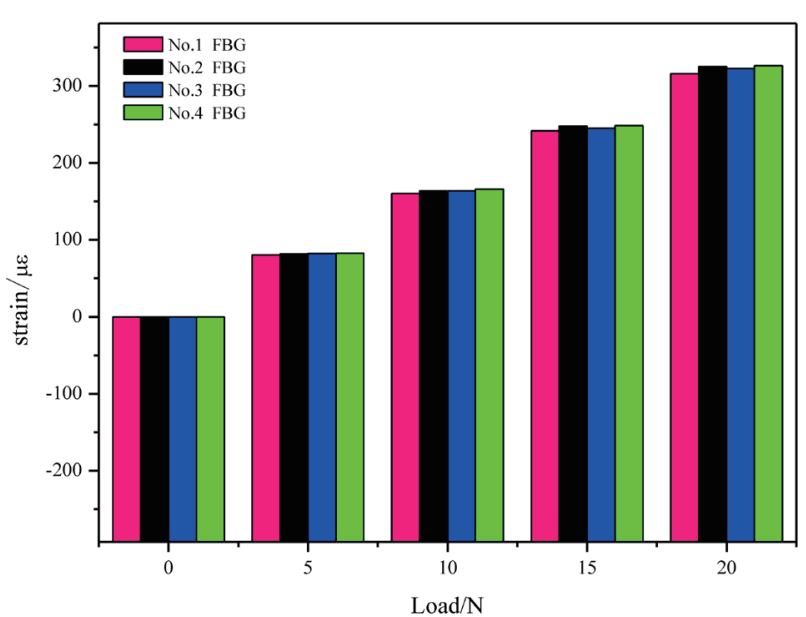

(b)

Fig. 10. (Color online) Statistical chart of FBG measurements. The stability of FBG strain measurement was excellent and the fluctuation of data was very small.

FBG data. The fluctuation rates of the strain under 5, 10, 15, and $20 \mathrm{~N}$ loading were $0.027,0.035$, 0.024 , and 0.033 , respectively, and the average value was 0.0307 . The maximum fluctuation rate of the strain gauge data was 0.092 , greater than that of the FBG sensors. Therefore, the stability of FBG technology for strain measurement is clearly superior to that of traditional resistance strain gauges. FBG sensors can avoid the errors caused by differences in the resistance, connecting wires, connection patterns of conductors, and so forth. Moreover, the optical parameters have high sensitivity.

\section{Numerical Simulation Analysis}

\subsection{Software overview}

ANSYS numerical analysis software is among the most powerful general finite element analysis software in the world. The software has a powerful nonlinear computing function and both preprocessing and postprocessing abilities. It can build models through basic primitives such as point-line-plane. It can also use Boolean operations to add, subtract, overlap, and paste geometric models. It is more suitable than other numerical simulation software for building complex models. ANSYS software contains many constitutive models, including Link (bar), Beam, Shell, Solid, and other models. It integrates the structure, fluid, heat, electromagnetism, and other factors organically, and is widely used in fields including transportation, aviation, mining, and power. 


\subsection{Model establishment}

The solid186 constitutive model was chosen to build a model of the uniform-strength beam. The model elements were high-order, three-dimensional, 20-node solid structure elements, which can better simulate irregular mesh elements with a secondary displacement mode. The uniform-strength beam had a variable cross section, with a large number of irregular elements appearing after meshing. The elasticity modulus was $210 \mathrm{GPa}$, Poisson's ratio was 0.3, and the density was $7800 \mathrm{~kg} / \mathrm{m}^{3}$.

The geometric model was established in accordance with the dimensions of the uniformstrength beam used in the calibration experiment: the geometric similarity ratio was 1:1, the length was $1200 \mathrm{~mm}$, the width of the loading section was $24 \mathrm{~mm}$, the width of the fixed end was $75 \mathrm{~mm}$, and the thickness was $5 \mathrm{~mm}$. The model was divided into 7977 elements and 16742 nodes with a $10 \mathrm{~mm}$ fixed edge length. The fixed end of the model was constrained by three directions of displacement. The load gradient was $5 \mathrm{~N}$ and four stages of loading were carried out, consistent with the calibration experiment.

\subsection{Analysis of results}

Figure 11 shows the surface displacement and strain of the uniform-strength beam numerical model under a load of $5 \mathrm{~N}$. The displacement of the beam exhibited gradient variation with a

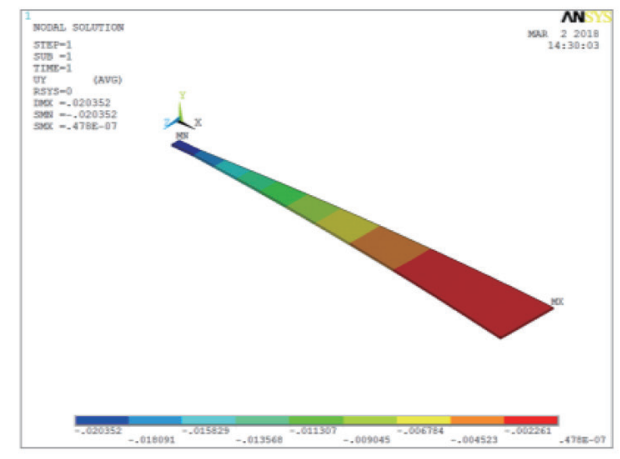

(a)

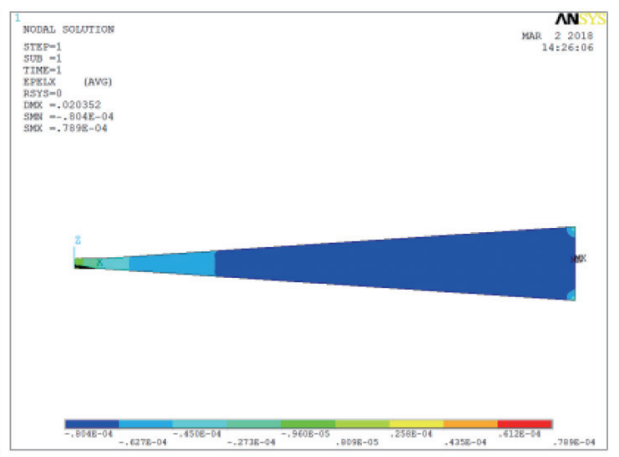

(b)

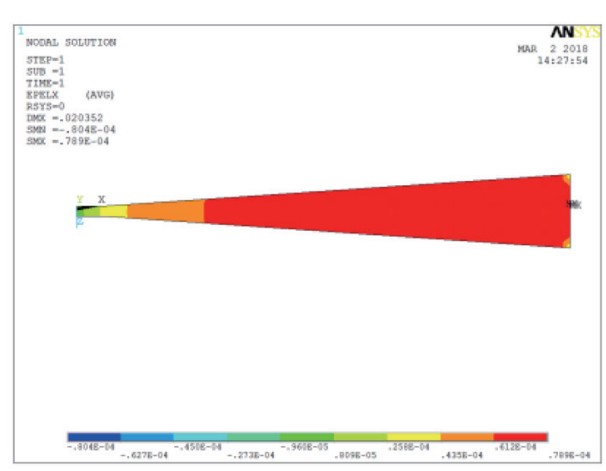

(c)

Fig. 11. (Color online) Surface displacement and strain of numerical model. The strain is positive on the upper surface and negative on the lower surface. (a) Upper-surface displacement under load of $5 \mathrm{~N}$, (b) lower-surface strain under load of $5 \mathrm{~N}$, and (c) upper-surface strain under load of $5 \mathrm{~N}$. 
maximum displacement of $0.02 \mathrm{~m}$ at the loading end and a minimum displacement of $0.002 \mathrm{~m}$ at the fixed end. As shown in Figs. 11(b) and 11(c), the surface strain was basically constant, the lower-surface strain was negative, and the upper-surface strain was positive. When the loading end of the uniform-strength beam was bent downward, tensile stress acted on the upper part of the neutral plane and compressive stress acted on the lower part of the neutral plane, consistent with the results of the above analysis. The surface strain of the beam was found to be $78.9 \mu \varepsilon$ under a load of $5 \mathrm{~N}$.

Figure 12 shows the surface strain distributions in the numerical model of the uniformstrength beam under loads of $5,10,15$, and $20 \mathrm{~N}$. The surface strains of the beam under the loads of $5,10,15$, and $20 \mathrm{~N}$ were $78.9,158,237$, and $315 \mu \varepsilon$, respectively. The deviation between the results of the numerical simulation, the theoretical calculation, and the calibration test was small, which shows that the calibration test is accurate and reliable. In fact, the theoretical results are not the true strains of the uniform-strength beam because the parameters used in the theoretical

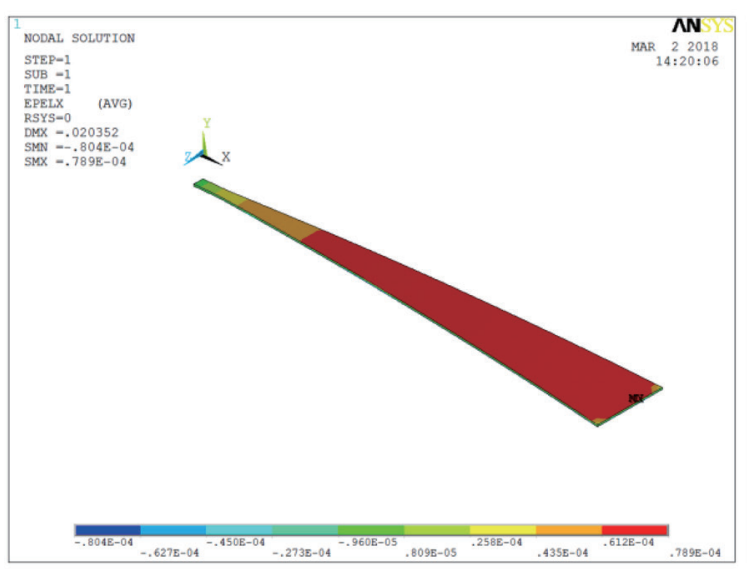

(a)

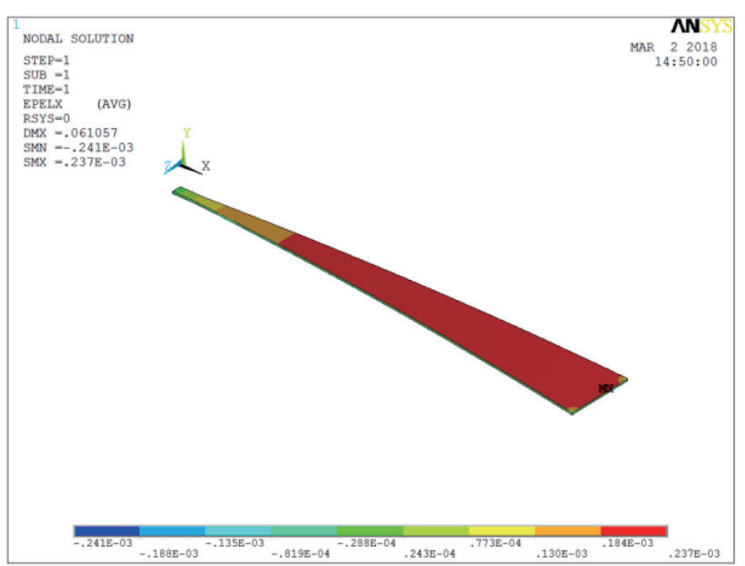

(c)

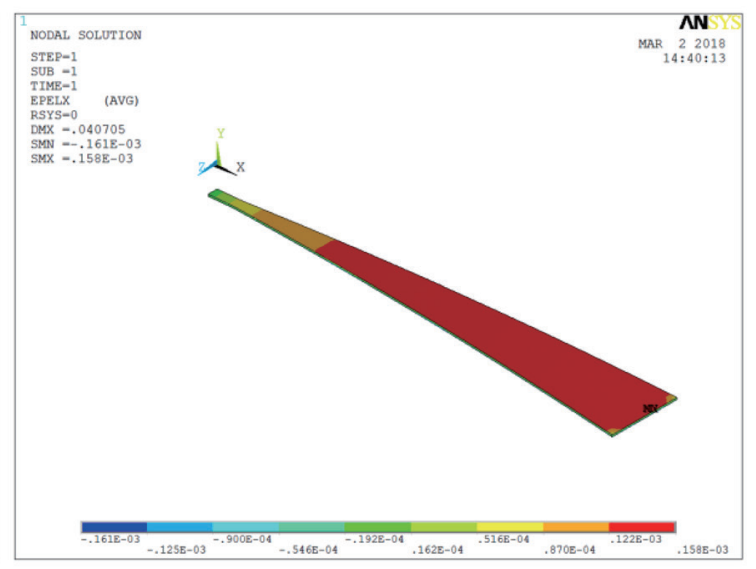

(b)

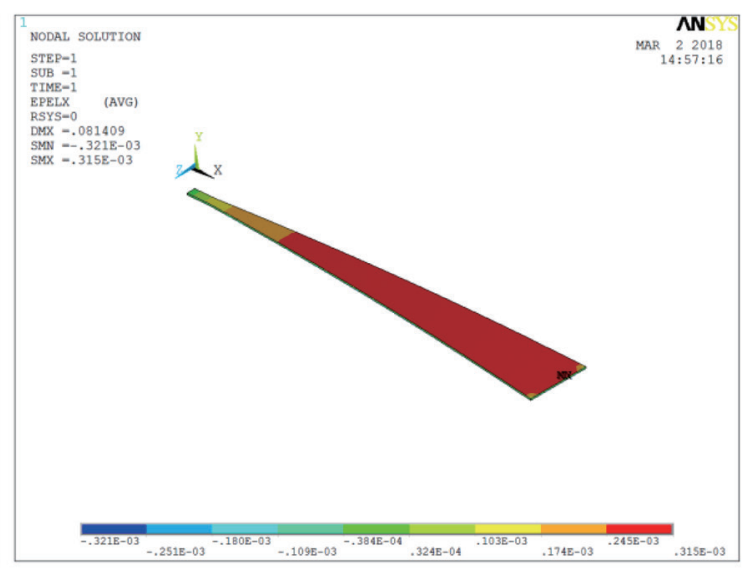

(d)

Fig. 12. (Color online) Strain under different loads obtained by numerical model. (a) Upper-surface strain under load of $5 \mathrm{~N}$. (b) Upper-surface strain under load of $10 \mathrm{~N}$. (c) Upper-surface strain under load of $15 \mathrm{~N}$. (d) Upper-surface strain under load of $20 \mathrm{~N}$. 
Table 3

Strain coefficients of optical fibers obtained from comprehensive analysis.

\begin{tabular}{lccc}
\hline Category & Diameter $0.1 \mathrm{~mm}$ & Diameter $0.7 \mathrm{~mm}$ & Diameter $0.9 \mathrm{~mm}$ \\
\hline Calibration test result & 0.041 & 0.044 & 0.047 \\
Numerical simulation result & 0.050 & 0.053 & 0.057 \\
Average value & 0.0455 & 0.0485 & 0.0520 \\
\hline
\end{tabular}

calculation, such as the length and thickness of the beam, also have measurement errors. Therefore, when calibrating optical fiber sensors, a variety of methods should be used simultaneously, such as using different kinds of sensors and carrying out a theoretical calculation and a numerical simulation. To ensure the reliability of the calibration results, we calibrated the strain coefficient of the distributed optical fibers a second time by using the numerical simulation results.

For optical fiber 1 with a diameter of $0.1 \mathrm{~mm}$, the average Brillouin frequency shifts under the loads of 5, 10, 15, and $20 \mathrm{~N}$ were 4.334, 8.350, 12.933, and $15.940 \mathrm{MHz}$, respectively, and the calculated strain coefficient was $0.05 \mathrm{MHz} / \mu \varepsilon$. For optical fiber 2 with a diameter of $0.7 \mathrm{~mm}$, the average Brillouin frequency shifts under the loads of 5, 10, 15, and $20 \mathrm{~N}$ were 5.324, 9.009, 13.999, and $17.756 \mathrm{MHz}$, respectively, and the calculated strain coefficient was $0.053 \mathrm{MHz} / \mu \varepsilon$. For optical fiber 3 with a diameter of $0.9 \mathrm{~mm}$, the average Brillouin frequency shifts under the loads of $5,10,15$, and $20 \mathrm{~N}$ were $6.254,10.965,15.276$, and $19.867 \mathrm{MHz}$, respectively, and the calculated strain coefficient was $0.057 \mathrm{MHz} / \mu \varepsilon$. The strain coefficients calculated by the numerical simulation were larger than the calibration test results. By comprehensively considering the two sets of results, the strain coefficients of the optic fibers with diameters of $0.1,0.7$, and $0.9 \mathrm{~mm}$ were determined to be $0.0455,0.0485$, and $0.0520 \mathrm{MHz} / \mu \varepsilon$, respectively (Table 3).

\section{Conclusions}

In this study, the strain test performances of BOTDA and FBG technologies were investigated by carrying out calibration tests on a uniform-strength beam and uniaxial compression tests on standard rock specimens. Strain gauges, DIC, BOTDA, and FBG techniques were comparatively analyzed, and the strain measurement performance, monitoring stability, system error, and strain coefficients of distributed optical fibers with different diameters were obtained. An auxiliary verification was carried out by ANSYS numerical simulation. The following conclusions were obtained.

(1) According to the test results, the measurement accuracy of the distributed optical fibers was $\pm 25 \mu \varepsilon$. That is, when the measured strain was less than $25 \mu \varepsilon$, the measured data is invalid and cannot be used to analyze practical problems. When the measured strain exceeded $25 \mu \varepsilon$, the strain was considered to be caused by the deformation of the measured object. When the frequency shift of the optical fiber was positive, the optical fiber was under tensile stress, and when the frequency shift was negative, it was under compressive stress. On the basis of this 
rule, the stress state of the measured object can be characterized using a distributed optical fiber.

(2) The relationship between the axial strain and time for the rock specimens was determined using an FBG sensor and DIC. The trend of the strain measured by the two methods was similar, especially during elastic compression. The fluctuation of the DIC measurement data was greater than that of the FBG sensor data, which indicates that the FBG measurement is more stable. The maximum fluctuation rate of the strain gauge data was 0.092 and that of the FBG data was 0.031 . The stability of FBG technology for strain measurement is clearly superior to that of traditional resistance strain gauges. The FBG sensor can avoid the errors caused by differences in the resistance, the connecting wires, and the connection patterns of conductors. Moreover, the optical parameters have high sensitivity.

(3) According to the calibration test results, the thicker the packaging material, the higher the sensitivity to stress. A thicker packaging material increases the contact area between the optical fiber and the object under test, makes the stress transfer more efficient, and improves the sensitivity of the optical fiber. To ensure the reliability of calibration results, the strain coefficients of the distributed optical fibers were calibrated a second time by using the numerical simulation results. The strain coefficients calculated by the numerical simulation were larger than the calibration test results. Considering the two sets of results, the strain coefficients of the optical fibers with diameters of $0.1,0.7$, and $0.9 \mathrm{~mm}$ were determined to be $0.0455,0.0485$, and $0.0520 \mathrm{MHz} / \mu \varepsilon$, respectively.

\section{Acknowledgments}

We are grateful for financial support by the Key Program of National Natural Science Foundation of China (Nos. 41027002, 51174280, and 51804244). Many thanks go to Professor J. Chiai for the guidance in this paper. We would also like to thank Dr. Zhang and Dr. Yuan for their valuable comments and suggestions for improving the manuscript.

\section{References}

B. Madjdabadi, B. Valley, and M B. Dusseault: Measurement 77 (2016) 54.

C. J. S. D. Matos, P. Torres, and L. C. G. Valente: J. Lightwave Technol. 19 (2001) 1206.

L. Liu, H. Zhang, and Q. Zhao: Opt. Fiber Technol. 13 (2007) 78.

4 S. T. Oh, W. T. Han, and U. C. Paek: Opt. Express 12 (2004) 724.

5 Y. Zhao, Y. Liao, and S. Lai: IEEE Photonics Technol. Lett. 14 (2002) 1584.

Y. Le, S. H. Lee, and O. L. Kon: FEBS Lett. 425 (1998) 367.

7 T. Horiguchi, T. Kurashima, and M. Tateda: IEEE Photon 1 (1989) 107.

8 D. Culverhouse, F. Farahi, and C. N. Pannel: Electron. Lett. 25 (1989) 913.

9 T. Horiguchi and M. Tateda: J. Lightwave Technol. 7 (2002) 1170.

10 T. Horiguchi, K. Shimizu, and T, Kurashima: J. Lightwave Technol. 13 (1995) 1296.

11 X. Bao, J. Dhliwayo, and N. Heron: J. Lightwave Technol. 13 (2002) 1340.

12 X. Bao, D. J. Webb, and D. A. Jackson: Opt. Lett. 19 (1994) 141.

13 W. Du, J. Chai, and D. Zhang: Int. J. Rock Mech. Min. Sci. 141 (2021) 1.

14 J. Chai, W. Du , Q. Yuan, and D. Zhang: Opt. Fiber Technol. 48 (2019) 84.

15 C. Hong, Y. Zhang, G. Li, M. Zhang, and Z. Liu: Sens. Actuators, A 285 (2017) 131.

16 H. Murayama and H. Igawa: Photonic Sens. 3 (2013) 355.

17 J. He, Z. Zhou, and O. Jinping: Mech. Syst. Sig. Process. 35 (2013) 84. 
18 Z. Zhou, X. Zhao, and C. Wang: Proc. SPIE 5 (2004) 2.

19 K. Kageyama, I. Ohsawa, and M. Kanai: Proc. SPIE 2 (2003) 205.

20 M. Iten, A. M. Puzrin, and A. Schmid: Proc. SPIE Int. Soc. Opt. Eng. 69 (2008) 33.

21 X. H. Jia, Y. J. Rao, and K. Deng : IEEE Photonics Technol. Lett. 23 (2011) 435.

22 A. Signorini, C. J. Oton, and F. D. Pasquale: J. Lightwave Technol. 34 (2016) 342. 